\title{
Study of Chinese Whole Books Reading Teaching in Senior High School From the Perspective of Instructional Interaction in Connectivist Learning Context
}

\author{
Liang Weiying ${ }^{1}$
}

\begin{abstract}
${ }^{1}$ School of Chinese Language and Literature, Beijing Normal University, Haidian, Beijing, China Corresponding author.Email:690094905@qq.com
\end{abstract}

\begin{abstract}
Connectionism is a learning theory in line with the characteristics of the information era, which can provide a new theoretical perspective for the research and practice of Chinese whole books Reading Teaching in senior high school. This study is designed to analysis Chinese whole books Reading Teaching in senior high school from the perspective of instructional interaction in connectivist learning contexts. According to cognitive engagement, the teaching process is divided into four levels: Operation interaction, wayfinding interaction, sensemaking interaction and innovation interaction. Based on the interaction characteristics of each level, this study puts forward corresponding teaching suggestions for Chinese whole books Reading Teaching in senior high school.
\end{abstract}

Keywords: Whole books reading, Reading Teaching, connectivism, instructional interaction

\section{从联通主义教学交互视角看高中语文整本书阅读教学}

\author{
梁蔚莹 1 , a
}

1 北京师范大学文学院, 海淀, 北京, 中国

a690094905@qq.com

\begin{abstract}
摘要
联通主义是符合信息化时代特色的学习理论, 能够为高中语文整本书阅读教学的研究与实践提供新的 理论视角。本研究通过联通主义教学交互视角审视高中语文整本书阅读教学, 按照认知参与程度由低 到高将教学过程分为操作交互、寻径交互、意会交互与创生交互四个层次, 并根据各个层次的交互特 征提出相应的高中整本书阅读教学建议。
\end{abstract}

关键词: 整本书阅读，阅读教学，联通主义，教学交互

\section{1. 引言}

近年来, 随着《普通高中语文课程标准 (2017 年版)》 的颁布, “整本书阅读”的概念也成为了语文教育界关注 的焦点, 围绕着整本书阅读的历史渊源、价值内涵、实 际操作, 学术界与语文教学一线展开了大量的研究与实 践, 也在整本书阅读建设上取得了客观的成就。可以说, 在语文改革的浪潮下, 整本书阅读在 理论与实践方面 已经有了一定的成就。然而, 在大量的研究成果之下, 整本书阅读的研究视野仍然失于狭窄。在“CNKI 中国知 网”对“整本书阅读”进行检索, 会发现 7.63\%的研究主题
都与“阅读教学”有关, 在所有主题中排名第二, 仅次于 “整本书阅读与研讨”主题。这体现出高中语文整本书阅 读的研究视野聚焦于语文教学一线之上, 而这些针对整 本书阅读的研究中, 鲜少根据系统的研究理论加以指导, 仅仅是一线教师的课堂实录。整本书阅读的研究, 需要 新的理论指导, 从符合 21 世纪时代特征的新视野出发, 重新审视高中语文整本书阅读的教学实践。联通主义作 为“数字时代的学习理论” [1], 以前瞻性的眼光对学习进 行解释分析, 可以被称为“面向未来教育的学习理论”。 本文以联通主义审视当前高中语文整本书阅读, 以期为 高中语文整本书阅读研究与实践提供新的思路与参考。 


\section{2. 联通主义理论及发展}

联通主义是一种适应于当前时代背景下社会结构 的学习理念。在学习观上, 联通主义认为学习即是连接 的建立和三个网络（神经网络、概念网络和外部社会网 络) 的形成[2]; 在知识观上, 它认为知识存在于连接中 [3]。在课程观上, 联通主义开发了开放、分布、学习者 定义、社会化和复杂的课程[4], 学习空间与学习形式更 加多样, 学习者自主选择参与学习形式, 课程内容由参 与者共同开发; 在教师观上, 教师被视作是课程的促进 者而非引领者, 负责影响与塑造网络 [5]; 在环境观上, 联通主义关注学生在复杂信息环境下个人学习环境与 个人学习网络的构建; 在交互观上, 联通主义将交互视 为学习的核心 [6]。

与之前的行为主义、认知主义以及社会建构主义学 习理论相比, 联通主义的理念与当前时代特征更为贴合, 它将神经学、认知心理学、脑科学、网络学等学说进行 融合, 关注的是在复杂的真实情境下, 模糊、无确定答 案的需集众人智慧才能解决的问题 [5]。在此基础上, 联 通主义突破了传统学习理论的时间与空间限制, 关注人 在网络交互中的终身学习; 学习的目的从掌握知识转变 为学会学习, 增强创造力; 学习主体不仅仅关注个体, 而且关注个人与群体的交互; 人及信息联通的媒介一 网络时代的价值被着重强调, 信息时代下的复杂网络能 真正把一切具有教育资源和关系联结起来, 构建立体的、 多维的、协同的, 多场合一的, 弹性而有序的完整的学 习系统[7]。

结合整本书阅读相关的理念, 整本书阅读关注学生 在与文本交流、与学生交流、与教师交流的过程中对于 文本意义的建构, 从而掌握阅读方法与阅读策略, 达到 思维的发展提升, 在此过程中, 教师更多地作为学生阅 读的促进者与学生讨论的组织者。整本书阅读能够提供 相对完整的文化场域 [8], 这无疑为学生提供了复杂模糊 的信息环境, 考验的是学生在整本书的复杂真实信息情 境中的思考问题、解决问题能力。不难看出, 整本书阅 读的理念与联通主义的学习理念均强调学生在复杂多 样的情境中掌握学习能力, 突出学生学习的主体性。以 联通主义的视角分析高中语文整本书阅读具有可行性, 并且很有可能碰撞出全新的火花。

\section{3. 联通主义教学交互模型下的高中语文整 本书阅读}

联通主义认为交互是学习发生的核心要素。“交互” 的定义从诞生以来就较为模糊, 20 世纪 70 年代交互概 念与远程通信结合, 被定义为双向通信 [9]以及指学生与 教师或教育机构成员之间的交流[10], 其后概念的定义 大多不超过“双向交流”的范畴。在针对联通主义的教学 交互模型研究中, 交互的本质被定义为“为了让学习者 达到学习目标, 学习环境中的主体间相互交流和相互作 用的过程”[11], 该研究在基于学习过程的会话模型[12] 建立的教学交互层次塔[13]之上, 建构了基于认知参与
度的联通主义学习教学交互模型。该模型将联通主义学 习中的教学交互按照 认知参与度从浅入深, 对学习者 的难度分为操作交互、寻径交互、意会交互和创生交互 四层。联通主义学习是四类交互作用下的螺旋式的知识 创新和网络扩展与优化的过程[14]。接下来将从联通主 义学习教学交互模型视角出发, 从四个层次分析整本书 阅读的教学实施。

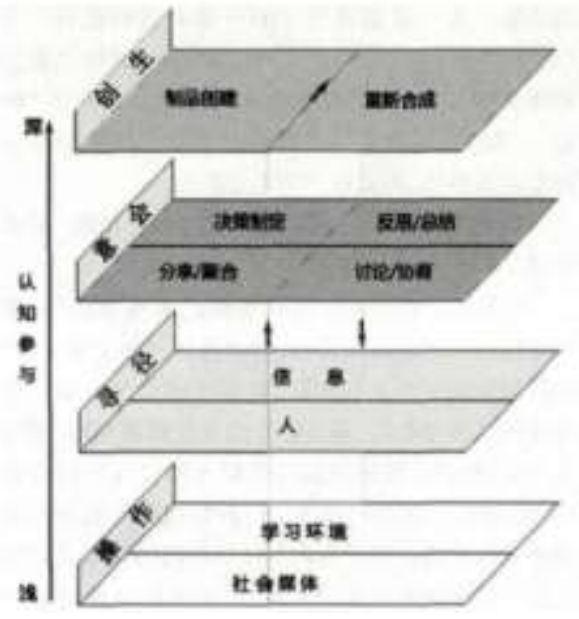

图 1-基于认知参与度的联通主义学习教学交互 分层模型[11]

\section{1. 操作交互}

操作交互指学习者为了开展学习与各种媒体和技 术环境之间的相互作用，即学习者与界面之间的交互。 在联通主义学习环境中, 操作交互的目的即建立交互空 间或者（个人与集体的）学习环境[11]当前的众多研究 通常将整本书阅读的学习环境仅仅局限于课堂内。但是 随着近年来 Web2.0 时代的来临以及教育技术的迅猛发 展, 高中语文整本书阅读的学习环境大可以突破封闭、 不流动的教室空间，而向更为开放流通的网络平台进行 拓展。中华人民共和国教育部在《教育信息化 2.0 行动 计划》中要求建设互联互通、开放灵活多级分布、覆盖 全国、共治共享、协同服务的国家数字教育资源公共服 务体系, 正是寻求利用社交媒体平台的建构来创设数字 时代的学习环境。在这种的开放、分享、协作、创造、 联通的学习环境中, 学生能够根据自身需求来个性化地 定制学习环境, 也就是由学生自己决定自己获取知识, 创造与分享知识的内容、方法与对象。

在高中语文整本书阅读与研讨的教学实践中, 师生 应明确, 仅仅发生在课堂领域内的阅读行为在广度与深 度上远远无法达到培养学生阅读素养的要求。整本书阅 读在课堂的发生, 更大程度上是播撒下了阅读的种子, 激发学生的阅读兴趣, 促使学生根据自身的阅读偏好去 设计自己的个性化阅读方案。因此, 在课堂阅读交流之 外, 教师还应该拓展更为广阔的网络交流平台, 利用网 络搜索引擎为学生提供更多的阅读辅助材料, 深化学生 的阅读理解, 而阅读辅助材料的多样性则为学生的自主 
选择提供了足够的空间。同读《红楼梦》, 学生可以选择 从小说中诗词的角度搜寻文献, 理解判词背后揭示的人 物命运, 也可以从小说中女性的角度出发, 以女性主义 的视角重新审视各式各样女性角色在时代背景下的际 遇与选择, “一千个读者就有一千个哈姆雷特”, 在课堂 上将片面的个人的见解当作普遍适用的权威知识灌输 给学生, 显然是不合适的, 利用媒体构造的广阔学习环 境让学生在大量的阅读中建立起自己的价值判断和审 美角度, 这对于学生在阅读中的思维进阶、文化传承与 审美塑造更具效果。此外, 教师也可以依托网络平台进 行线上交互, 除常规的学习网站、论坛以外, 微信、腾 讯 QQ 等交流软件也可以灵活地运用起来, 作为平时的 交流平台, 引导学生在轻松的阅读氛围中随时进行交流 分享协作，创造与获取新的知识。

\section{2. 寻径交互}

寻径交互指学习者为了在学习环境 (或者交互空间) 中进行定向而与环境的符号、标记和线索相互作用, 可 以联通知识流通的管道 [15]。它包括人际交互 (个体与 个体、个体与群体、个体与网络之间的交互), 又包括人 与内容之间的交互, 维持学习之间的连接最主要的方式 是实现与信息的直接连通和与人的联通 [11]。

寻径交互在整个教学交互过程中起到了十分重要 的作用, 它上承操作交互所构建的个性化学习网络, 下 接意会交互的交流讨论与决策环节, 寻径交互的顺利进 行能够保证整个学习过程的运行顺畅。在整本书阅读的 学习中, 学生一方面需要与信息建立联系, 即积极阅读 整本书以及围绕整本书的各种辅助性阅读材料, 另一方 面也需要与人建立联系, 即积极构建共同阅读的学习小 组。学习共同体能够为学生的学习提供良好的氛围, 在 一个集体学习的环境内, 学生的思维碰撞才能产生更多 有价值的创生品。在高中语文整本书阅读教学中, 构建 共同讨论的学习环境, 引导学习共同体围绕一个问题进 行共同学习, 是值得推荐的, 这种共同学习在许多专题 教学实践中得到体现 $[16,17]$ 。教师可以积极推动学生组 成学习小组, 或是在交流平台中主动地就阅读体验进行 观点、情感的分享, 积极表达困惑与解答别人的困惑, 在主动寻径、参与式寻径与帮助他人寻径的过程中建立 起属于自己的信息网络与人际网络, 获得激励与支持, 以进行进一步的交互学习。

\section{3. 意会交互}

意会交互指学习者为了获得对不确定、复杂和变化 环境中的问题和信息的响应而投入参与的过程, 它发生 在学习者内部和学习者之间的连接建立与观点形成的 过程中, 是一种内隐的模式识别和信息搜寻。意会交互 方式包括聚合与分享、讨论与协商、反思与总结和决策 制定四类[11]。在意会交互的层面上, 学生能够真实地 进行学习观点的汇集加工, 进行观点的交流碰撞, 对交
流进行反思总结，并且对于阅读与交流中的问题进行决 策。在这个过程中。学生与学习内容之间的交互更加深 入。以高中语文整本书阅读学习为例, 学生在进行讨论 交流的过程中, 也就做到了对于阅读文本的个性化重构, 达到了对于文本内容整体感知之上的理解、应用、分析、 评价, 认知参与层次由浅及深。

落实在高中语文整本书阅读的教学中, 教师需要树 立联通主义教学理念, 不再是支配性论断的灌输者, 也 不再是课堂的主导者, 而是学习环境建构、学习网络搭 建的协助者。教师应该在整本书阅读与研讨教学中营造 出平等开放的交流空间，引导学生自由地对阅读内容进 行交流讨论，畅所欲言，从思维的碰撞中诞生出智慧的 结晶, 而对于这些从自主学习、交流合作中产生的收获, 学生也会对其更具认同感。美国的青少年名著计划就以 研讨会的形式进行, 教师不过多地进行教学或解释, 只 是负责维持研讨会的“会话”状态[18], 而所有参加者, 包括主席在内, 都保持一种虚心开放的态度, 也做好接 受讨论结果的准备。他们敞开心胸, 虚心以待, 准备接 纳新的看法。对于崭新的观念或事物，不固执抵抗，但 也不会不假思索的全盘接受[19]。在这种交流研讨的过 程中, 学生需要将所有的信息加以汇总、筛选, 在讨论 中解决问题、深化思考, 阅读的知识也就从意会交互中 诞生并且强化, 与此同时, 学生的批判性思维得到锻炼, 对于阅读的兴趣也进一步提升。

\section{4. 创生交互}

创生交互是一个知识创造和生长的过程, 它包括为 了进一步的联通而单独或集体地表达和呈现新的观点、 解决方案、理论模型, 这种表达方式包括学习制品的创 建和重新合成 $[11]$ 。创建学习制品以事实、概念、过程、 程序或原则的方式对知识进行转录, [20], 如创建系统、 深入、逻辑的博客、文章或 其他形式的资源 建立观点 丰富, 赋予学习价值的视频、音频等 [14]; 重新合成作 为创建制品的一种, 则更强调从已有的知识信息中创造 或修改新内容 [21], 如利用已有的开放资源重新组合, 表达新的观点和想法 将碎片化的内容, 按照一定的规 则整合，呈现新的含义 [14]。创生交互是认知参与的最 顶层, 需要学生在与学生以及学习内容的交互中创造生 长知识, 在这个过程中, 学生的学习网络也进一步得到 优化, 促进新一轮交互学习的进行。

在高中语文整本书阅读的教学中, 创生交互即生成 学习成果的过程, 这个学习成果可以分散性地存在于每 一次的交流讨论中, 也可以通过教师系统性的组织规划, 形成具有体系的学习成果, 如李显晖老师在专题教学中 从确定选题到共同研讨, 最后帮助学生形成论文[22]。 除此以外, 还可以组织各种各样的学习成果制作, 如博 客写作、绘本、微信公众号文章、网页制作等, 这些学 习成果能够通过学生前期形成的学习网络进行共享, 学 生也能够在对他人成果的学习中继续生成知识。在创生 交互中, 教师应注重及时反馈, 也就是关注过程性评价。 尊重学生按照自己的学习兴趣选择目标主题、搜集材料、 
交流讨论、生成成果的一系列过程中的多样性, 探寻学 习目标与学习结果的对应性, 提炼学习过程存在的问题。 在此基础上, 充分促进教师、学生不同主体的互动, 发 挥学习共同体的作用, 促进学生学习过程的有效展开 [23], 真正落实“为学习而评价”。

\section{REFERENCES}

[1] Siemens, G.(2005).Connectivism:A learning theory for the digital age. International Journal of Instructional Technology and Distance Learning, 2(1):3-10.

[2] Siemens, G. (2011). Orientation: Sensemaking and wayfinding in complex distributed online information environments. Aberdeen: University of Aberdeen Doctoral dissertation.

[3] Rumelhart, D, Posner, M. (1989). The architecture of mind: A connectionist approach. In Foundations of Cognitive Scienc. Cambridge: MIT Press:133-159.

[4] Siemens,G.(2013).Massive Open Online Courses: Innovation in Education?[DB/OL]. Retrieved on July 12, 2014 from https://oerknowledgecloud. org/sites/oerknowledgecloud. org/files/ pub_PS_OERIRP CHI. pdf.

[5] Wang, Z. J., Chen, L. (2014)The Learning Theory of Connectivism and its Latest Development .Open Education Research, 20(05):11-28. (in Chinese)

[6] Downes, S. (2012). Connectivism and Connective Knowledge: Essays on Meaning and Learning Networks. [DB/OL]. National Research Council Canada. Retrieved on June 9,2014 from http:// www. downes. ca/ files/ books/ Connective_Knowledge-19 May 2012. pdf.

[7] Wang, Y. M., Zhu, Z. T. (2006). From connectionism to Connectivism: a new orientation of learning theory. China Educational Technology, (03):5-9. (in Chinese)

[8] Wu, X. X. (2017). Reading the whole book, improving Core Competence of Chinese subject as a whole. Language Teaching in Middle School, (01):11-14. (in Chinese)

[9] Bääth, J. A. (1979). Postal two -way communication in correspondence education, ICCE Newsletter, 9, 1, s: 2-9.

[10] Daniel, J. S.\& Marquis, C. (1979). Interaction and independence: getting the mixture rights, Teaching at a Distance, vol.14, spring: 29-44.

[11] Wang, Z. J.,Chen, L.(2015). Theory Framework
Building of Instructional Interaction in Connectivist Learning Context. Open Education Research, 21(05):2534. (in Chinese)

[12] Laurillard,D.(2001).Rethinking university teaching: A conversational framework for the effective use of learning technologies(2nd).London: Routledge.

[13] Chen, L. (2004). A Hierarchical Model for Student and Teacher Interactionin Distance Learning. China Distance Education, (05):24-28+78. (in Chinese)

[14] Wang, Z. J.,Chen, L.(2016). Instructional Interaction Pattern and Method Analysis in cMOOCs. China Educational Technology, 2016(02):49-57. (in Chinese)

[15] Siemens, G. (2006).Knowing knowledge [DB/OL].Retrieved on June 19 , 2014 from www.knowingknowledge.com.

[16] Yang, Y (2017). Teaching arrangement of reading the whole book of Les Miserables. Bulletin of Chinese Language Teaching, 2017(10):9-12. (in Chinese)

[17] Li, Y. H. (2019). Study on the same topic: teaching reading of the whole book "wandering". Chinese Language Learning, 2019(06):14-19. (in Chinese)

[18] Li, J. (2008). The Junior Great Books Program and significance for China. Minzu University of China, Beijing. (in Chinese)

[19] MortimerJ. A., translated by Lin, Q. B. (2003).How to speak. How to listen. Haikou: Nan Hai Publishing Co.:186.

[20] Clark,R., \&Chopeta,L.(2010).Graphics for learning: proven guidelines for planning, designing, and evaluating Visuals in training materials, Hoboken: John Wiley\& Sons.

[21] Belshaw,D.(2013). First draft of Mozilla' s Web Literacy standard now available[DB/OL]. Retrieved on July 26,2015 from http:/dougbelshaw.com/blog/2013/04/26/first-draft-ofmozillas-web-literacy-standard-now-available/.

[22] Li, Y. H. (2019). The journey of exploration and discovery: Thematic teaching of the whole book reading, Shanghai: Shanghai Education Publishing House.

[23] Zheng, G. M.,Li, Q.(2015). Strengthening the Accumulation of Excellent Poetry and Classics: The Transition of Value Orientation of Chinese Curriculum. Educational Research, 36(11):98-102. 